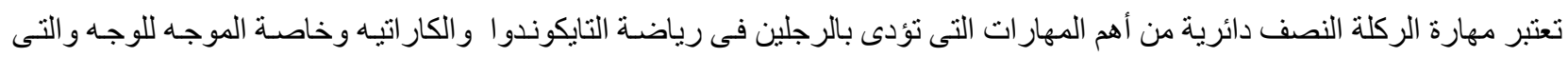

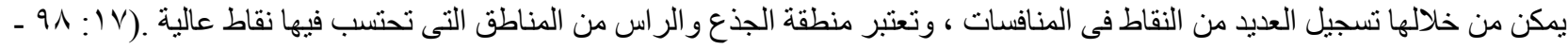
$($ Yr: $1 \cdot)($ rVO: 10) (1)

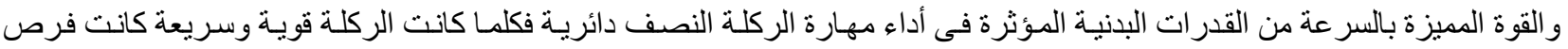

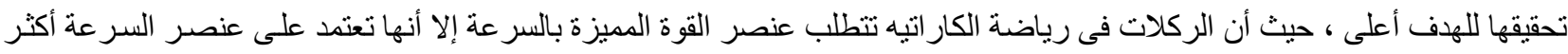

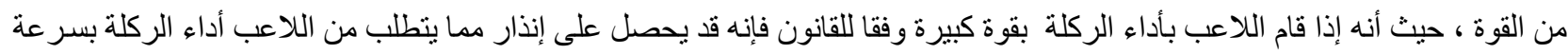

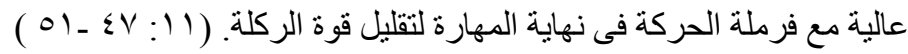

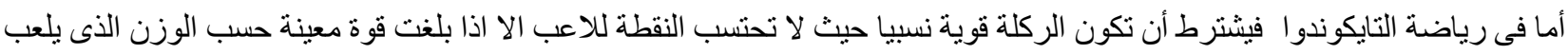

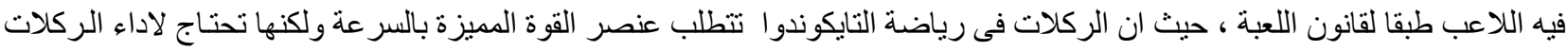

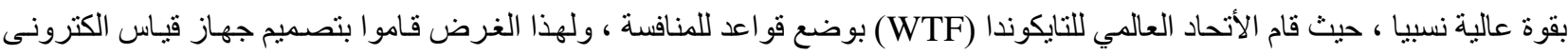

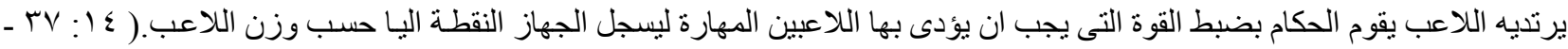

ولتوليد القوة المميزة بالسر عة لمهارة الركلة النصف دائرية لابد من تحديد العضلات المشاركة فى الأداء من خـلال تحديد نسبة مسـاهمة

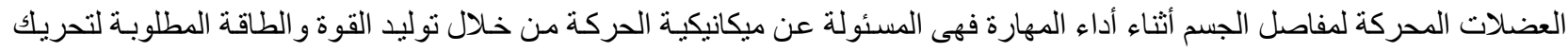

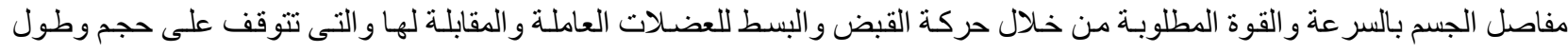

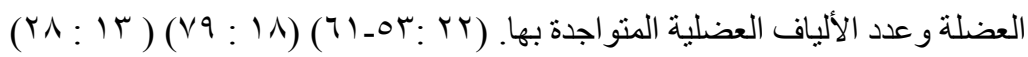
فالعضلات هى التى تتتج القوة والتى تتسبب فى تغيير حركة الجسم فهى المصدر الوحيد القادر على إنتاج القوة الداخلية وبدونها نكون

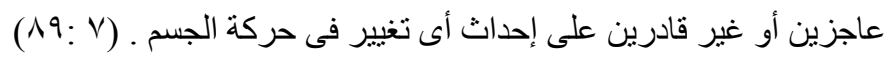

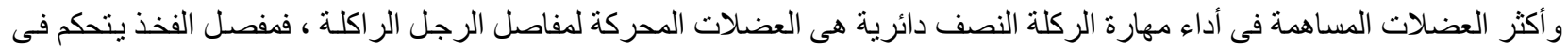

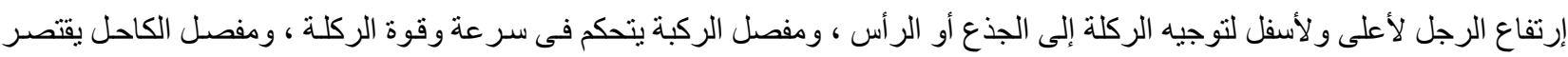

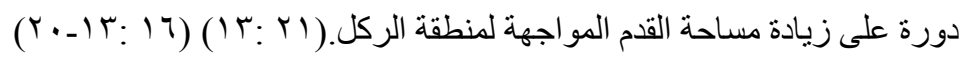

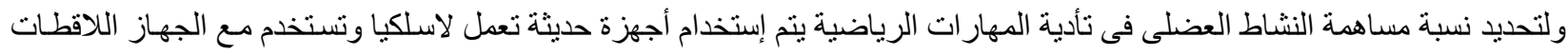

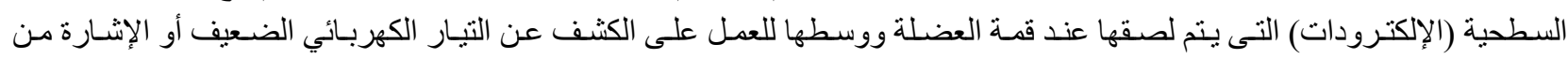

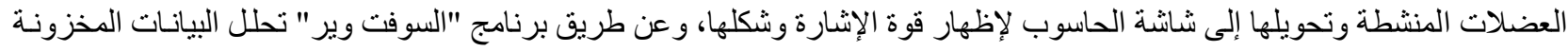

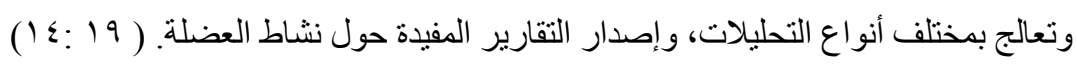

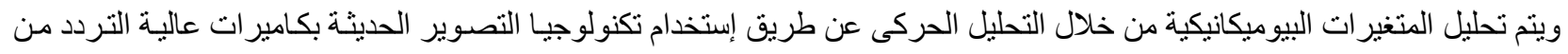

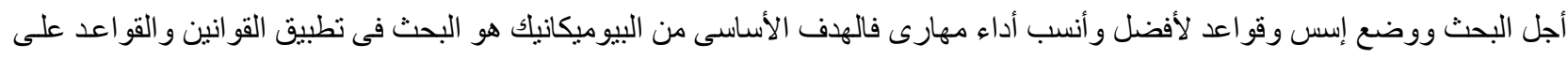

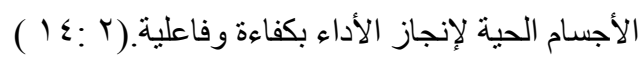

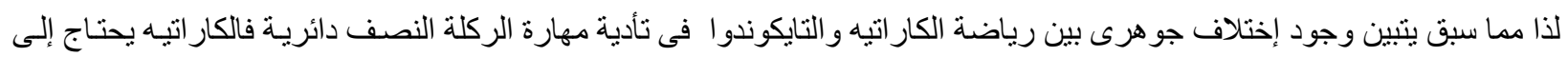

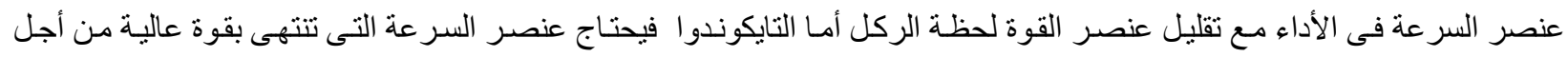

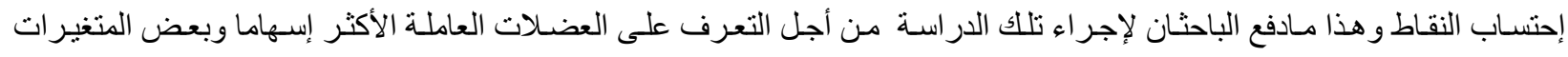

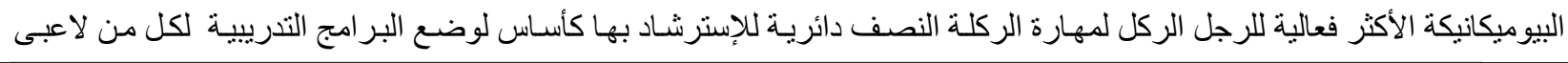


الكار اتيه و التنايكوندو التحسين تكنيك الأداء المهارى .

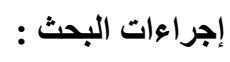

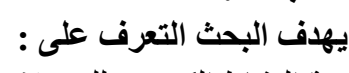

مقارنة النشاط الكهربى للعضلات وبعض البعض المتغير ات البيو ميكانيكة لأداء الركلة النصف دائرية بين لاعبى الكار اتيه و التايكوندو التحسين

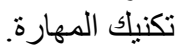

$$
\text { فروض البحث :- }
$$

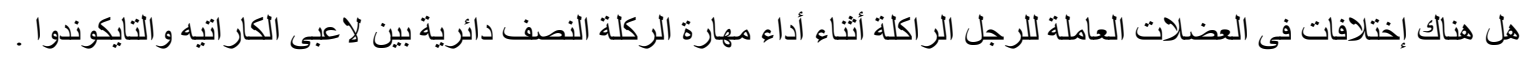

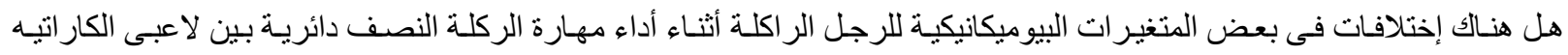

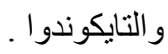
- إستخدم الباحثان المنهج الوصفى لمناسبته لطبيعة البحث.

$$
\text { • مجالات البحث: }
$$

تم إختيار عينة البحث بالطريقة العمدية وعددهم ستة لاعبين من المستوى العالى ثلاث من لاعبى الكار اتيه وثلاثة تايكوندا و التى تنتو افر

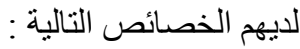
-

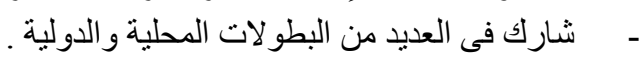

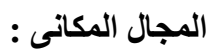

تم إجر اء الدر اسة الأساسية فى معمل الميكانيكا الحيوية بكلية التربية الرياضية بنين - جامعة الأسكندرية .

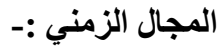

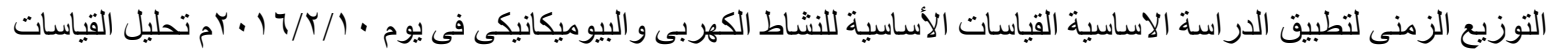

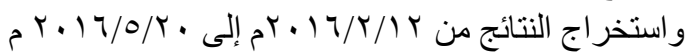

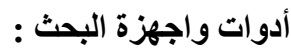

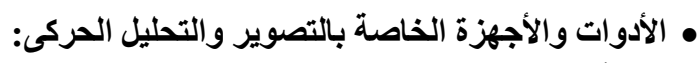

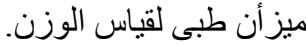

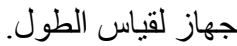

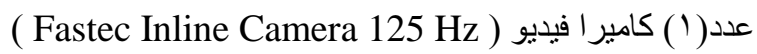
برنامج ( Max Traq ) لتسجيل المحو لات من الكامير على جهاز لاب توب ماركة (Hp) (Hp) -

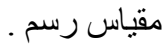
عدد ( 0 ) بكرات شريط لاصق لتحديد نقاط ومفاصل الجسم .

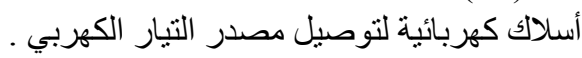

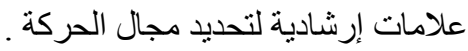

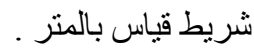

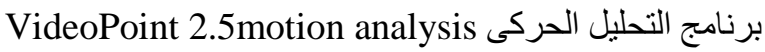
الأدوات والاجهزة الخاصة بقياس النشاط الكربى للعضلات:

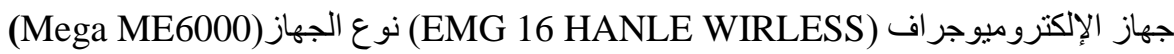
ـ كامير ا تردد · ب ك / ث من نوع (Canon DVC320W). ـ الكترودات من نوع skin tact، كحول، قطن، ماكينات حلاقة، شريط طبى لاصق.

$$
\begin{aligned}
& \text { الاراسة الأساسية : } \\
& \text { خطوات إجراء الاراسة: - مالة } \\
& \text { تم اجر اء الدر اسة على ثلاثة مر احل رئيسية : } \\
& \text { أولا: مرحلة التجهيز: - }
\end{aligned}
$$




\section{صبحى حسونة حسونة}

محمد أحمد عبد الفتاح زايد

ا. تم تحديد المتغير ات التى سيستخرجها الباحثًان من خلال أجهزة القياس المستخدمة التى تعمل فى تز امن واحد لمر احل

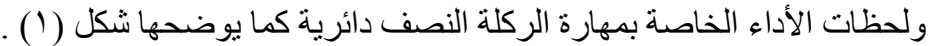
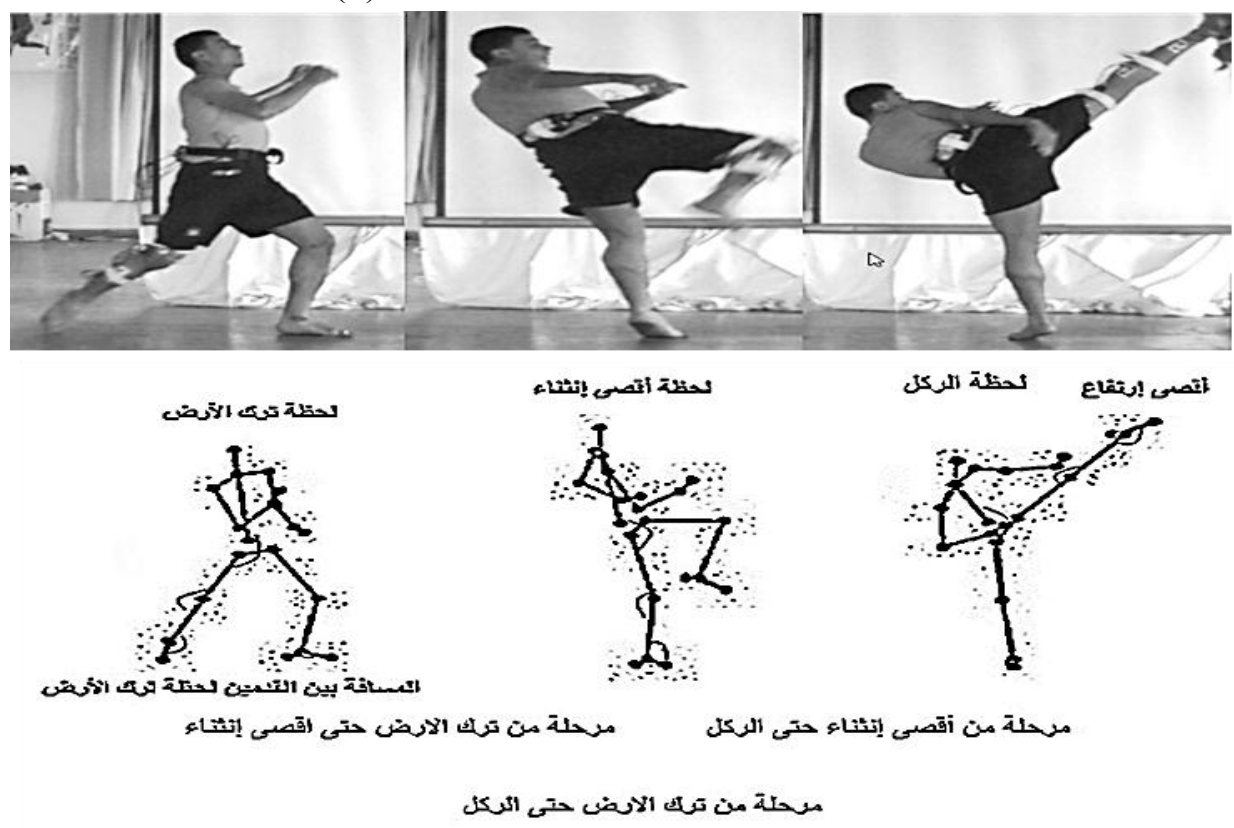

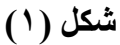

يوضح مراحل ولحظات أداء مهارة الركلة النصف دائرية

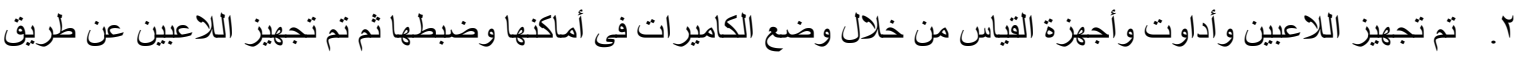
وضع الإلكترودات على العضلات عن طريق حلاقة الثعر ووضع الكحول قبل وضع الإلكترودات على العضلات وذللك

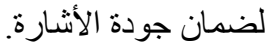

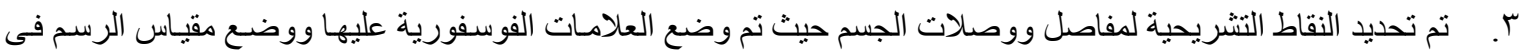

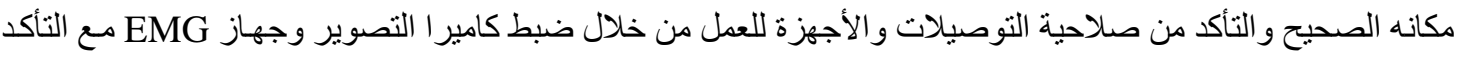
من إستقبال الأشارة من الجهاز بصورة لاسلكية.

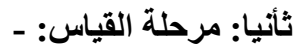

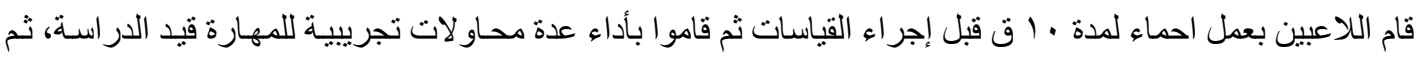

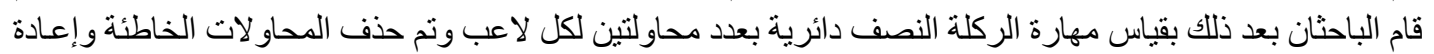

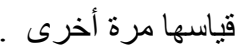

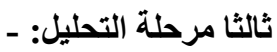

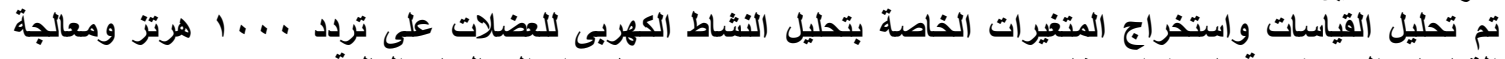

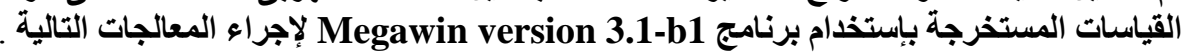

$$
R M S v a l u e[I]=\sqrt{\frac{\sum_{i=n}^{n+N-1}\left|D a t a_{R a w}[i]\right|^{2}}{N}}
$$

-Where: I = index of RMS data - مؤشر جذر متوسط مربع البيانات

•i = index of raw data - مؤشر البيانات الخام

- $\mathrm{N}$ = number of data points in

RMS calculation n = [1, N+1, 2N+1, ...]. عدد نقاط البيانات في حساب مربع متوسط الجذر) 


\section{صبحى حسونة حسونة}

محمد أحمد عبد الفتاح زايد

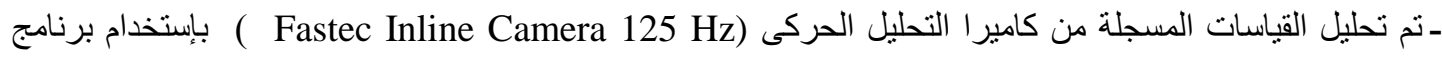

VideoPoint 2.5motion analysis

لحظات التحليل المحددة فى وضع خطى كما يوضح الثكل رقم (1) (1).

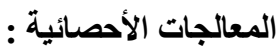

إجريت المعالجات الأحصائية التى تتناسب مع طبيعة هذا البحث باستخدام برنامج 21.0 SPSS ميث نم تطبيق الطرق

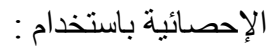

$$
\text { - }
$$

أختبار ت Intenendent - Samples T Test.

\begin{tabular}{|c|c|c|c|c|c|c|c|c|}
\hline \multicolumn{4}{|c|}{ 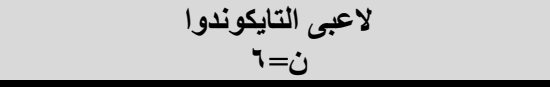 } & \multicolumn{4}{|c|}{ لاعبى الكاراتيه } & \multirow[b]{2}{*}{ المتغيرات } \\
\hline التقلطح & الألتواء & الألحراف ال المعيارى & المسابي & معامل & الألتواء & المعيارى الأنحر & المسابي & \\
\hline & \multicolumn{8}{|c|}{ المرحلة من ترك الارض حتى أقصى إنثناء } \\
\hline $1 . \leqslant Y_{-}$ & $\cdot r \cdot-$ & $\because \cdot r$ & .19 & Y.०V & $1 . \mathrm{Vr}$ & $\because \cdot r$ &.$r \Delta$ & زمن \\
\hline Y.Y $\varepsilon_{-}$ & $\because \cdot 1-$ & $\leqslant . \leqslant 0$ & $1 V . \leqslant \varepsilon$ &.$V Y_{-}$ &..$\leqslant \varepsilon$ & 7.00 & 19.9 & العضلة الأليية الكبرى لرجل الركل \\
\hline$r . \cdot 7$ & 1.00 & $1.9 \leq$ & $9 . \cdot 7$ & $1.0 \Lambda_{-}$ & .97 & 0.07 & $17 . r \mu$ & العضلة المستقيمة الفخذية الرباعية \\
\hline$\cdot \cdot 1$ & $\cdot .99$ & Y.r. & $\varepsilon .7 r$ & .07 & 1.00 & $1 . v \cdot$ & A.YY & العضلة المستقيمة الوحشية الرباعية \\
\hline $1.99-$ & $.1 Y_{-}$ & $1.9 r$ & 7.r & $\cdot r \cdot-$ & $\because 9 r$ & 7.07 & $1 \cdot . r V$ & العضلة المستقيمة الأنسية الرباعية \\
\hline r.IV- & $\cdot r^{q}$ & $1 . \leqslant \varepsilon$ & $17 . \wedge 7$ & $r . r$ & $1.7 \mathrm{~V}$ & $\varepsilon .11$ & $1 \% . \wedge 7$ & العضلة ذات الرأسين الفخذية الخلفية \\
\hline$Y_{.} \cdot q_{-}$ & $. r \leq$ & $7 . V$. & 10.97 & $r_{.} \cdot r_{-}$ & $\because 9-$ & $r . \wedge 9$ & $\vee . \wedge 9$ & العضلة القصبية الأمامية لرجل الركل \\
\hline $1 . r 7-$ & .17 & $r .74$ & 17.90 & $\cdot r r_{-}$ & $\because v \cdot-$ & $1 . Y Y$ & $\wedge .1 \mathrm{~V}$ & الجزء الخلفي لعضلة الساق لرجل الركل \\
\hline$\cdot .9 q$ & .997 & Y.\&. & $1 Y . \leqslant \Lambda$ & $1.9 \leqslant-$ & $\because \cdot r$ & $0 . V Y$ & 17.00 & العضلة النعلية لرجل الركل \\
\hline
\end{tabular}

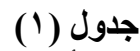

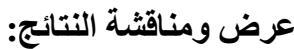

\begin{tabular}{|c|c|c|c|c|c|c|c|c|}
\hline .99 & $\because Y \theta_{-}$ & $\because \cdot r$ & $\because 1$. & r.A9 & 1.01 & $\because \cdot r$ &. $.1 Y$ & زمن \\
\hline $1 . \wedge Y_{-}$ & $\because \wedge \mathrm{V}$ & $9 . \wedge 7$ & $10.9 \mathrm{~V}$ & $. .9 \leq-$ & $\cdot . \wedge 0$ & $0 . r$. & $1 \varepsilon_{.}$. & العضلة الأليية الكبرى لرجل الركل \\
\hline $1 . \mu 1-$ & $\cdot . \tau_{-}$ & V.YI & $r \cdot . V \leqslant$ &. $.0 \mathrm{~V}$ & $\cdot \wedge^{\wedge}$ & r.OT & $Y \leqslant . Y_{0}$ & الرباعية الرؤوستئل الرجة الفخلية الركل \\
\hline $1 . \leqslant 0$ &. .00 & $0 . V V$ & $11 . \mathrm{VV}$ & $\because .9$ &.$\diamond V_{-}$ & $1 . V \leqslant$ & $1 \cdot .70$ & الرباعية الرؤوس لرجلة الوجل الركيّة \\
\hline
\end{tabular}
أولا : عرض نتائج الدالات الأحصائية للبحث :

الالالات الإحصائية الخاصة بمتغيرات البحث لمراحل ولحظات أداء مهارة الركلة النصف دائرية للاعبى الكاراتيه و التايكوندوا

المرحلة من أقصى إنثناء حتى الركل 
صبحى حسونة حسونة

محمد أحمد عبد القتاح زايد

\begin{tabular}{|c|c|c|c|c|c|c|c|c|}
\hline $1 . \wedge \varepsilon$ & $\because \wedge \varepsilon-$ & $\varepsilon . \mu r$ & $1 \% . .$. & $1 . \cdot \varepsilon$ & $\because 97$ & r.IV & $1 V .10$ & الرباعية الرؤوس المستقيمة الأنسية الرجلة \\
\hline 1.AT- & $\because \vee \Lambda_{-}$ & $\bullet .0 \Lambda$ & 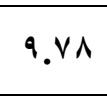 & $\cdot \wedge{ }_{-}$ & $\because \wedge$ & 0.11 & $1 . .11$ & العضلة ذات الرأسين الفخذية \\
\hline r. & 1.94 & $7 . \cdots$ & 1.10 & $Y_{.} \Delta V_{-}$ &. .17 & $\varepsilon \_$\& & $\Lambda . r q$ & العضلة القصبية الأمامية لرجل \\
\hline $1.87_{-}$ & 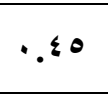 & $\curlyvee . \wedge \wedge$ & $q . \cdot r$ & $r_{.} \cdot 1-$ & $\because v$ &.$\wedge \wedge$ & $0 . M r$ & الجزء الخلفي لعضلة الساق) لرجل الركل \\
\hline $1 . Y Y_{-}$ & $. .71-$ & $r . \mu$. & $11.0 r$ & $.1 V_{-}$ & $\because \varepsilon Y$ & $0 .+r$ & $1 . .1 \%$ & العضلة النعلية لرجل الركل \\
\hline
\end{tabular}

المرحلة من ترك الارض حتى الركل

\begin{tabular}{|c|c|c|c|c|c|c|c|c|}
\hline. .17 & $\cdot . V r$ & $\because \cdot r$ & $\cdot . r^{9}$ & Y.. 9 & $\overline{r . Y I}$ & $\because .0$ & 9.49 & زمن \\
\hline Y.VV- & $\because .7$ & $7.7 \leqslant$ & $1 \Lambda_{.} \cdot V$ & Y.r.I- & $\because \cdot 0_{-}$ & $0.7 \mathrm{~V}$ & IV.Or & العضلة الأليية الكبرى لرجل الركل \\
\hline 5.19 & $1 . \leqslant 0$ & $1 . \leqslant 0$ & $1 \varepsilon . r q$ & $.01-$ & .01 & $\varepsilon . Y V$ & 11.91 & الرباعية الرؤوستئية لرجل الفذذية الركل \\
\hline 1.01 & $1 . r v$ & $1 . r 0$ & V.T & $r \leqslant-$ & •.rT_ & .91 & $9 . \cdot r$ & الرباعية الروؤلة المستقيمة الوحشل الركلة \\
\hline צ.74- & .11 & 1.01 & 9.14 & $\cdot V \leqslant-$ & .94 & ๕.74 & $|Y . V|$ & 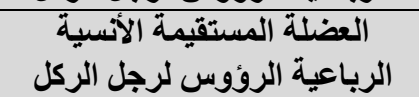 \\
\hline $1 . r T-$ & .00 & r. $\leqslant$ & $14.1 \leqslant$ &.$T V_{-}$ & .71 & $\varepsilon . r q$ & IY.VY & الخلة ذات الرأسين الفخذية \\
\hline r.... & $\because \varepsilon \varepsilon$ & r.Tr & $1 Y . \leqslant 4$ & r.. O_ & $\ddots^{4}$ & $\varepsilon . V$ & $\Lambda_{.} \cdot v$ & العضلة القصبية الأمامية لرجل \\
\hline 1.9. & $\because \varepsilon$ & $1 . \leqslant 0$ & $|r . \leqslant|$ & בצד. & - & $\cdot 94$ & $v .1 v$ & الجزء الخلفي لعضلة السداق \\
\hline Y.OY & $\because \cdot V_{-}$ & Y.7. & 11.70 & r.1.- & $\cdot . r$ & $0 . Y Y$ & $1 r . \wedge 9$ & العضلة النعلية لرجل الركل \\
\hline
\end{tabular}


صبحى حسونة حسونة

محمد أحمد عبد الفتاح زايد

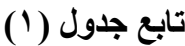

الدلالات الإحصائية الخاصة بمتغيرات البحث لمراحل ولحظات أداء مهارة الركلة النصف دائرية للاعبى الكاراتيه والتايكوندوا

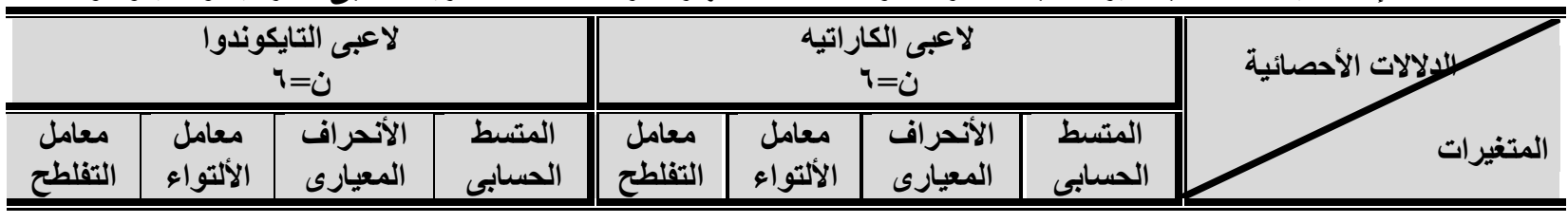

لعظة ترك الارض

\begin{tabular}{|c|c|c|c|c|c|c|c|c|}
\hline $1 . v 1-$ & $\cdot \wedge \Lambda_{-}$ & $\because \bullet$ & צ'T. & $\because \leqslant 1-$ & $\cdot \varphi^{\wedge}$ & $\because \cdot 7$ & $\because \vee \wedge$ & المسافة بين القدمين فى وضع \\
\hline $1 . r \varepsilon_{-}$ &.$r \varepsilon$ & 7.97 & 174.10 & $1.7 \%$ &. .44 & $r . \leqslant r$ & 109.91 & زاوية الفخذ لرجل الركل \\
\hline Y.79- & 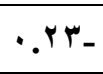 & IT.rY & 144.71 & 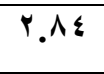 & 1.14 & $r . \cdot \varepsilon$ & $1 \leqslant 0.10$ & زاوية الركبة لرجل الركل \\
\hline r. $\left\{r_{-}\right.$ & . & $9.7 \varepsilon$ & $101 . r T$ & Y.OY &.$r_{\text {L }}$ & 15.79 & 10V.Tr & زاوية الكاحل لرجل الركل \\
\hline r.vq & 1.17 & .91 & r.tr & r.qY & 1.09 & $\cdot . r$. & r.rT & محصلة السرعة لنقطة الكاحل \\
\hline $1 . \Lambda_{-}$ & $\cdot \leqslant V_{-}$ &. .09 & $r . \leqslant Y$ & צ & $\because r$. & $\because 10$ & $r$. & ثحصلة كمية الحركة لمركز \\
\hline $.97-$ & $\cdot Y \varepsilon$ & 0.74 & $\leq Y . Y_{O}$ & r.vq & 1.14 & r.. q & $r \varepsilon . q V$ & محصلة القوة لمركز ثقل \\
\hline
\end{tabular}

لحظة أقصى إنثناء

\begin{tabular}{|c|c|c|c|c|c|c|c|c|}
\hline 9.7. & $\because .1 V_{-}$ & V.YY & IYY.TV & $1 . Y \Lambda_{-}$ & ?... & $\overline{V . Y T}$ & $1 \mathrm{IT1.7V}$ & زاوية الفخذ لرجل الركل \\
\hline $1.7 r_{-}$ & $\because \varepsilon \cdot-$ & $r_{.} \cdot v$ & A५.0. &..$V r$ & $\because 9 V_{-}$ & Y.19 & $90.7 \mathrm{~V}$ & زاوية الركبة لرجل الركل \\
\hline$\cdot . \vee \Lambda_{-}$ & 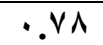 & $\varepsilon .07$ & $1 \leqslant Y_{.}$. & r.09 & 1.01 & $11^{1} 1$. & $1 \times 0.0$. & زاوية الكاحل لرجل الركل \\
\hline $1 . \cdots$ & $\because 0$ & $\cdot .94$ & $1 \cdot . r Y$ & 1.09 & - & .79 & $\Lambda . r 1$ & محصلة السرعة لنقطة الكاحل \\
\hline $1 . r \mu$ & $\because \cdots$ & $\because 4$ & \&.M & $r . \wedge$. & $1 . \wedge \cdot-$ & $\because$ vo & ץ. & محصلة كمية الحركة لمركز \\
\hline$\cdot r r$ & $1 . \cdot 1-$ & $\neg . \wedge \varepsilon$ & $0 \leqslant . r \wedge$ & r.09 & $1.51-$ & $\Lambda . \wedge \varepsilon$ & $\varepsilon \cdot . r$ & محصلة القوة لمركز ثقل \\
\hline
\end{tabular}

لحظة الركل

\begin{tabular}{|c|c|c|c|c|c|c|c|c|}
\hline $1 . r 0_{-}$ & $.0 \varepsilon_{-}$ & $\overline{Y .} \leqslant \varepsilon$ & 1.0 .70 & $\overline{C 1 . r V}$ & $\cdot \varepsilon q_{-}$ & $0 .$. & $11 Y .1 \mathrm{~V}$ & زاوية الفخذ لرجل الركل \\
\hline$\cdot .{ }^{\top}$ & $\because .1 \mathrm{~V}$ & 1.20 & IVV.TV &. $.1 Y$ & $1 . r T$ & $T . T_{T}$ & $17 \% .0$. & زاوية الركبة لرجل الركل \\
\hline 1.0 .1 &. $.1 \varepsilon$ & $1 \cdot . r 1$ & ITY.Y. & $\because \cdot r$ & .01 & $\varepsilon . \mu_{\Lambda}$ & $170 .$. & زاوية الكاحل لرجل الركل \\
\hline$T . Y \varepsilon_{-}$ & $\cdot . Y \varepsilon_{-}$ & $\cdot . v \cdot$ & E. Yo & $1 . \mathrm{VA}$ & $\cdot . \wedge \cdot$ & $\cdot . \leqslant 1$ & $1.2 \wedge$ & محصلة السرعة لنقطة الكاحل \\
\hline..$\leqslant \varepsilon$ & .70 & $0 . V \mu$ & $1 \Lambda . \varepsilon$. & r.00 & $1 . \leqslant r$ & 0.44 & $10 . \Gamma \varepsilon$ & محصلة كمية الحركة لمركز \\
\hline$\varepsilon .09$ & $r_{.} \cdot r$ & TV乏.VA & $904 . r 0$ & r.vo & 1.00 & $1 \Lambda \cdot . M r$ & T.T.T. & محصلة القوة لمركز ثقل \\
\hline r.Y.. & $\because .1 \mathrm{~V}$ &.$\cdot r$ & 1.71 & T.YT- &.$\cdot r$ & $\because .7$ & 1.79 & إرتفاع نقطة الكاحل لرجل \\
\hline
\end{tabular}

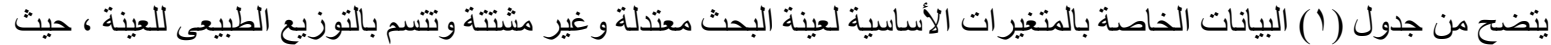

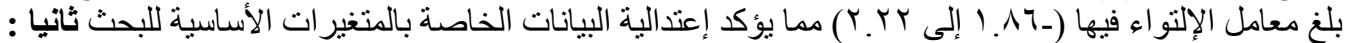

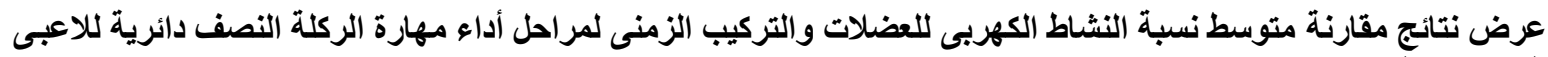

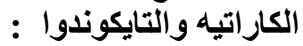




\section{صبحى حسونة حسونة}

محمد أحمد عبد الفتاح زايد

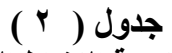

المقارنة بين لاعبى الكاراتيه والتايكوندوا فى متوسط نسبة النشاط الكهربى للعضلات والتركيب الزمنى لمراحل أداء مهارة الركلة النصف دائرية النفيربة

\begin{tabular}{|c|c|c|c|c|c|c|c|c|}
\hline \multirow{2}{*}{ قالمسة (ت) } & \multirow{2}{*}{ متوسين } & \multicolumn{2}{|c|}{$\begin{array}{c}\text { التايكوندوا } \\
7=0\end{array}$} & \multicolumn{2}{|c|}{ نالك= } & \multirow[b]{2}{*}{ وحدة القياس } & \multirow{2}{*}{ المعالجات } & \multirow[b]{2}{*}{ 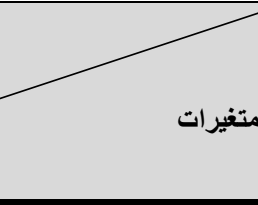 } \\
\hline & & المعيارى المع & 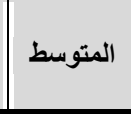 & المعبارى الأحرا & المتوسط & & & \\
\hline$* * r . V$. & 9.9 & $\because \cdot r$ &. .19 & 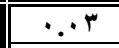 &..$r_{0}$ & \multirow{3}{*}{ 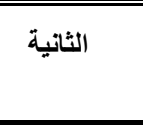 } & \multicolumn{2}{|c|}{ زمن مرحلة ترك الأرض حتى أقصى إنثناء } \\
\hline $1 . r r$ & $\because \cdot r$ & $\because \cdot r$ & $\because 1$ & $\because \cdot r$ & $.1 Y$ & & \multicolumn{2}{|c|}{ زمن مرحلة أقصى إنثناء حتى الركل } \\
\hline$* * 4 . \xi$ & $\because \cdot 1$ & $\because \cdot Y$ & $\because .99$ & $\because .0$ &. .47 & & \multicolumn{2}{|c|}{ زمن مرحلة ترك الأرض حتى الركل } \\
\hline$\because q$ & 1.01 & $\varepsilon . \leqslant 0$ & $1 V . \leq \varepsilon$ & 7.00 & 19.4 & \multirow{24}{*}{ 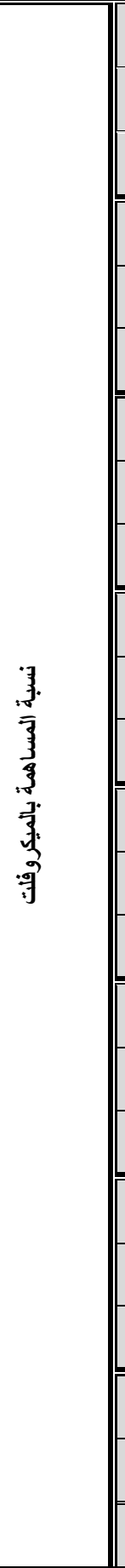 } & مرحلة ترك الأرض حتى إنثاء & \multirow{3}{*}{ لرضلة الأليية الكبرى } \\
\hline$\because \wedge$ & $\varepsilon .7$ & $1 \cdot . r 0$ & 11.97 & o.r. & $1 \leq .$. & & مرحلة أقصى إنثناء حتى & \\
\hline$\because 1$ & $\because 0 \leqslant$ & $7.7 \leq$ & $11 . \cdot v$ & $0.7 \mathrm{~V}$ & IV.Or & & مرحلة ترك الأرض حتى الركل & \\
\hline $\begin{array}{l}r .9 \\
* 1\end{array}$ & $\mathrm{~V} .1 \mathrm{~V}$ & $1.9 \leq$ & 9.97 & 0.04 & $17 . \mathrm{rr}$ & & مرحلة ترك الأرض حتى أنصى & \multirow{3}{*}{ الرضذية المستقيمة الريبة الرئية } \\
\hline$\ddot{i}$ & $1 . \leqslant \varepsilon$ & $\varepsilon .90$ & rY.Ar & r.or & $r \leqslant . r_{0}$ & & مرحلة أقصى إنثناء حتى & \\
\hline $\begin{array}{l}r .4 \\
* 4 \\
\end{array}$ & $\varepsilon .0 r$ & $1 . \leqslant 0$ & $1 \leq . r q$ & $\varepsilon . r V$ & 11.91 & & مرحلة ترك الأرض حتى الركل & \\
\hline $\begin{array}{l}r . \dot{ } \\
* r\end{array}$ & r.7. & r.ru & $\varepsilon .74$ & $1 . v \cdot$ & A.Y & & مرحلة ترك الأرض حتى أقصى & \multirow{3}{*}{ 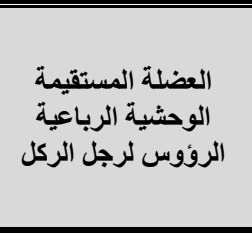 } \\
\hline 1.0 & r.0 & $r .09$ & 17.19 & $1 . v \varepsilon$ & $1 . .70$ & & مرحلة أقصى إنثاء حتى & \\
\hline $\begin{array}{l}r \cdot \dot{ } \\
* 1\end{array}$ & $1 . r \mathrm{~N}$ & $1 . r 0$ & $v .7 \varepsilon$ & 9.91 & $q . \cdot r$ & & مرحلة ترك الأرض حتى & \\
\hline $\begin{array}{l}1.4 \\
0\end{array}$ & $\varepsilon .0$ & $1.9 r$ & r. & 7.07 & $1 . . r v$ & & مرحلة ترك الأرض حتى أنقاء & \multirow{3}{*}{ الرؤوسية الرأرباعية المستيمة } \\
\hline $\begin{array}{c}1.7 \\
1\end{array}$ & r.v. & r.rr & $1 \leq . \leq \varepsilon$ & r.IV & 10.10 & & مرحلة أقصى إنثناء حتى & \\
\hline $\begin{array}{l}1 . v \\
v\end{array}$ & $r . \leqslant 0$ & $1 . * 1$ & 9.47 & צ.74 & $|Y . V|$ & & مرحلة ترك الأرض حتى & \\
\hline $\begin{array}{l}1.7 \\
1 \\
\end{array}$ & r... & $1 . \leqslant \leq$ & $17 . \wedge 7$ & \&.11 & Ir.人T & & مرحلة ترك الأرض حتى أنقاء & \multirow{3}{*}{ الفخذية الخلفية ذات الرجلن } \\
\hline$\because \wedge$ & $r_{.} \leqslant 0$ & o.or & $v .74$ & 0.11 & $1 . .11$ & & مرحلة أقصى إنثناء حنى & \\
\hline$i^{r}$ & $\because \varepsilon r$ & r. $\leq \varepsilon$ & $1 r .1 \leq$ & $\varepsilon . r q$ & IY.VY & & مرحلة ترك الأرض حتى & \\
\hline $\begin{array}{l}Y .0 \\
* 0\end{array}$ & $\Lambda_{.} \cdot v$ & ૫.V. & 10.97 & r.^q & $\vee . \wedge q$ & & مرحلة ترك الأرض حتى إنتى & \multirow{3}{*}{ الأمامية لرجلة القصبية الركل } \\
\hline 1. & r. 10 & r. $1 \leq$ & $7 . Y \leq$ & $\varepsilon . \varepsilon V$ & $\Lambda . r q$ & & مرحلة أقصى إنثاء حتى & \\
\hline $\begin{array}{l}1.9 \\
* v \\
\end{array}$ & $\varepsilon . r q$ & r.Tr & $1 Y_{.} \leq 7$ & $\varepsilon . V$ & $\Lambda_{\cdot} \cdot \vee$ & & مرحلة ترك الأرض حتى & \\
\hline $\begin{array}{l}. \varepsilon \\
* * \varepsilon \\
\end{array}$ & A.vq & r.Tr & 17.90 & I.rY & $\wedge .1 \mathrm{~V}$ & & مرحلة ترك الأرض حتى إنتى & \multirow{3}{*}{ الساق (السماتية) لرجل الرضلة } \\
\hline$i$ & $r_{.} \cdot \varepsilon$ & $\varepsilon . \wedge q$ & $v . r q$ & $\because \wedge \wedge$ & O.rY & & مرحلة أقصى إنثناء حتى & \\
\hline $\begin{array}{l}\Lambda_{.} \wedge \\
* * \wedge \\
\end{array}$ & $7 . r \leq$ & $1 . \leqslant 0$ & $\mid r . \leqslant 1$ & $\theta^{9 r}$ & V.IV & & مرحلة ترك الأرض حتى & \\
\hline 1.4 & $r .01$ & Y. \&. & Ir. $\{1$ & $0 . V Y$ & 17.00 & & مرحلة ترك الأرض حتى إنتى & \multirow{3}{*}{ العضلة النعلية لرجل } \\
\hline$\because$ & $\because 1$. & r.lr & $1 . . r r$ & $0 . r$ & $1 . .1 \mathrm{r}$ & & مرحلة أقصى إنثناء حتى & \\
\hline $\begin{array}{l}\because 9 \\
\varepsilon \\
\end{array}$ & $r . Y \leq$ & ז. . . & 11.70 & $0 . Y Y$ & $1 r . \wedge 9$ & & مرحلة ترك الأرض حتى & \\
\hline
\end{tabular}

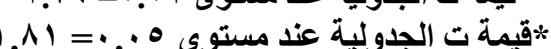




\section{صبحى حسونة حسونة}

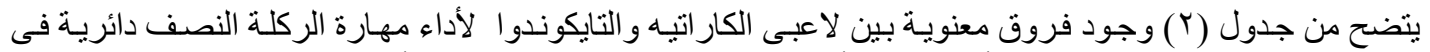

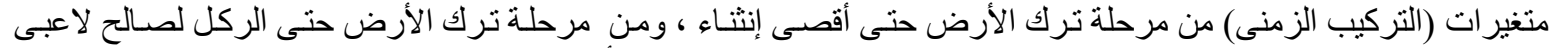

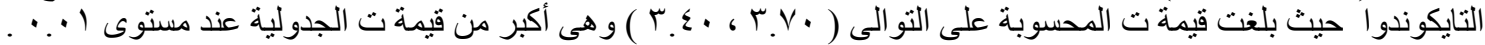

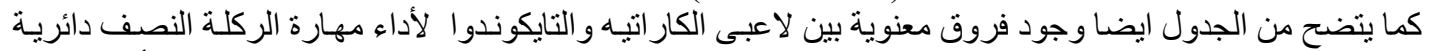

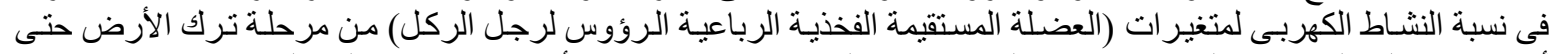

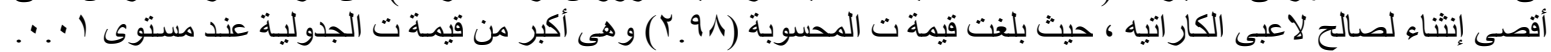

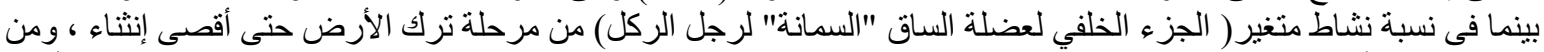

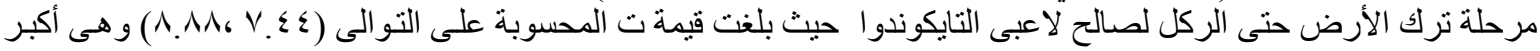

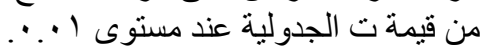

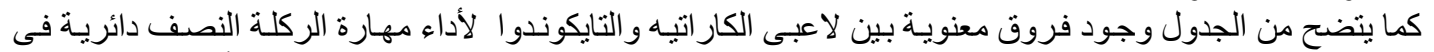

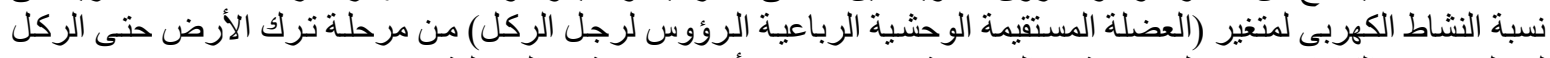

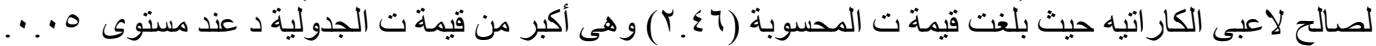

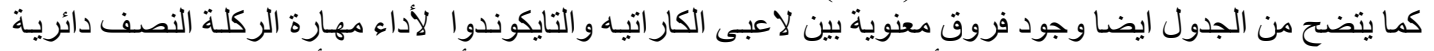

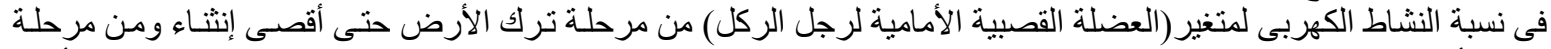

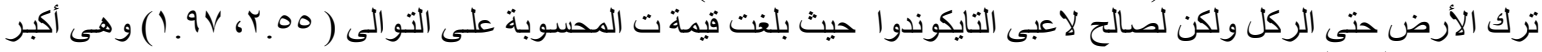

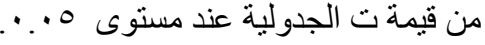




\section{صبحى حسونة حسونة}

محمد أحمد عبد الفتاح زايد

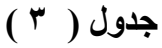

المقارنة بين لاعبى الكاراتيه والتايكوندوا فى متوسط بعض المتغيرات البيوميكانيكية اثناء مراحل أداء مهارة الركلة النصف دائرية

\begin{tabular}{|c|c|c|c|c|c|c|c|c|}
\hline \multirow{2}{*}{ قالمسلة (ت) } & \multirow{2}{*}{ متوسطين } & \multicolumn{2}{|c|}{ نإيكندو } & \multicolumn{2}{|c|}{ ن ألكاراتيه } & \multirow{2}{*}{ القياس } & \multirow[t]{2}{*}{ المعالجات } & \multirow{2}{*}{ متغيراد } \\
\hline & & 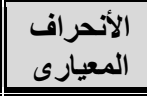 & المتوسط & 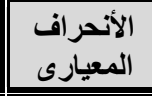 & المتوسط & & & \\
\hline$* * 0.71$ & $.9 \mathrm{~V}$ & $\because \cdot \varepsilon$ & $\cdot .74$ & $\because 4$ & $\because \vee \wedge$ & 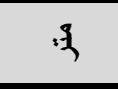 & ضوضع الأرتكاز & المسافة بين القدمين \\
\hline$* Y .{ }^{*}$ & $\curlyvee . \wedge \mathrm{V}$ & 8.97 & $174 . \wedge 0$ & $r . \leqslant r$ & 109.91 & \multirow{9}{*}{ 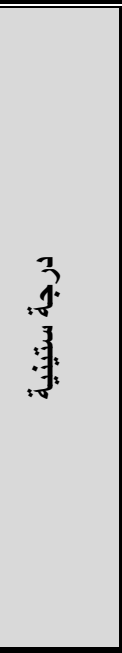 } & لحظة ترك & \multirow{3}{*}{ زاوية الفذذ لرجل } \\
\hline$* Y .10$ & $9 .$. & V.rq & IYY.TV & V.rr & $1 \mathrm{Tl} .7 \mathrm{~V}$ & & لحظة أقصىى & \\
\hline$* * Y . \wedge V$ & $7.0 Y$ & Y. $\leqslant \xi$ & 1.0 .70 & $\theta .$. & $11 Y .1 \mathrm{~V}$ & & لحظة الركل & \\
\hline ז & $9 . \cdot v$ & IT.r. & 174.71 & r.. \& & $1 \leqslant 0 . v 0$ & & لكظة ترك & \multirow{3}{*}{ زاوية الركبة لرجل } \\
\hline$* * v .0$ & $9.1 \mathrm{~V}$ & $r . \cdot V$ & ᄉร.०. & r.17 & $90.7 \mathrm{~V}$ & & لحظة أقصىى & \\
\hline$* * 4.0 Y$ & $9 . \wedge \mathrm{V}$ & 1.10 & IVV.rV & T.YV & $17 \% .0$. & & لحظة الركل & \\
\hline .94 & ד. & 9.7 ६ & $101 . \mathrm{rr}$ & 15.79 & 10V.TY & & لحظة ترك & \multirow{3}{*}{ زاوية الكاحل لرجل } \\
\hline$\cdot .^{\wedge}$ & 7.0 & $£ .07$ & $1 \leqslant \varphi .$. & 14.1. & $1 \times 0.0$. & & لحظة أقصىى & \\
\hline$\cdot . T Y$ & Y.A. & $1 \cdot . r 1$ & 17Y.Y. & $\varepsilon . \mu \wedge$ & $170 .$. & & لحظة الركل & \\
\hline $1 . \cdot r$ & $\cdot$. & .91 & $r .7 r$ & $\cdot r \cdot$ & T.r & \multirow{6}{*}{ 裹 } & لكظة ترك & \multirow{3}{*}{ لنقطة الكاحلة الربرعل } \\
\hline$* * \varepsilon . Y V$ & $r . \cdot r$ & .94 & $1 \cdot . r Y$ &. .79 & $\left.\Lambda .{ }^{\prime}\right)$ & & لحظة أقصىى & \\
\hline$* * \Lambda . r r$ & Y.VA & $\because \mathrm{V}$. & \&. Yo & $\because \leqslant 1$ & $1 . \leqslant 1$ & & لحظة الركل & \\
\hline 1.21 & 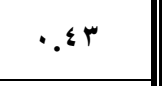 &. .09 & $r . \leqslant r$ & .10 & r... & & لحظة ترك الأرض & \multirow{3}{*}{ لمركز ثقلة اللرجل } \\
\hline$* Y . V 1$ & $\cdot 94$ &. .4 & צ. & $\because \mathrm{V}^{\circ}$ & T. & & لحظة أقصىى & \\
\hline$\because .11$ & $\because \mathrm{V}$ & $\because \leqslant 1$ & $1 . \varepsilon \Lambda$ & -.$\leqslant Y$ & $1 . r 1$ & & لحظة الركل & \\
\hline$* * Y . q \vee$ & $v .{ }^{\prime} \wedge$ & 0.74 & $\leqslant Y . Y_{0}$ & r.. 9 & $r \leqslant .9 v$ & \multirow{3}{*}{ 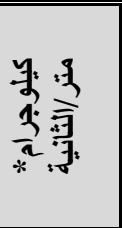 } & لكظة ترك & \multirow{3}{*}{ الحركة لمركز ثمثلة } \\
\hline$* * r .10$ & 1E.r & $\neg . \wedge \varepsilon$ & A & $\wedge . \wedge \varepsilon$ & $\varepsilon \cdot . r$ & & لـظة أقصى & \\
\hline .97 & $r . .7$ & $0 . v r$ & $1 \wedge . \varepsilon$. & 0.14 & $10 . r \varepsilon$ & & لحظة الركل & \\
\hline$* Y_{\text {. }} \leqslant V$ & ris.ro & YYะ.^D & $V \leqslant \Lambda .7 \Lambda$ & $17 \varepsilon .4$ & 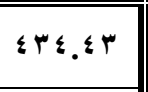 & \multirow{3}{*}{3} & ل لحظة ترك & \multirow{3}{*}{ لمركز ثقلّة الرجل } \\
\hline .91 & 1 & YIT.9v & $0.0 . \wedge \vee$ & $1 \leqslant 4 . \cdot \varepsilon$ & $019 . \leqslant V$ & & لحظة أقصىى & \\
\hline$* Y . .7$ & $r 0 . . .0$ & $r V \varepsilon . V A$ & 904.40 & $I A \cdot . r Y$ & Y.r.r. & & لحظة الركل & \\
\hline$* * Y . \wedge \wedge$ & $\because \cdot 1$ & $\because \cdot 4$ & 1.71 & $9^{4}$ & 1.79 & $\mathfrak{l}$ & \multicolumn{2}{|c|}{ إرتفاع نقطة الكاحل لرجل الركل } \\
\hline
\end{tabular}

يتضح من جدول(؟َ) وجود فروق معنوية بين لاعبى الكار اتيه و التايكو ندوا لأداء مهارة الركلة النصف دائرية فى متغير (المسافة بين

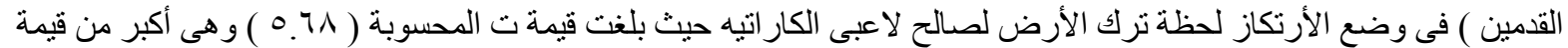

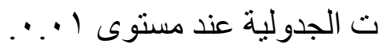




\section{صبحى حسونة حسونة}

كما يتضح وجود فروق معنوية بين لاعبى الكار اتيه و التايكو ندوا لحظة أقصى إنثاء ولحظة الركل فى متغير ( زاوية الفخذ لرجل الربل

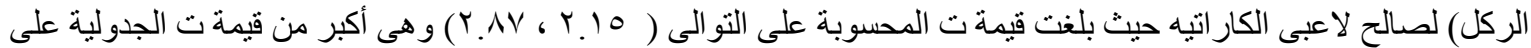

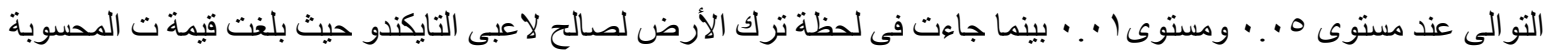

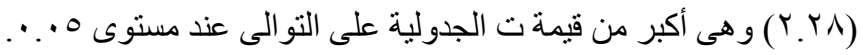

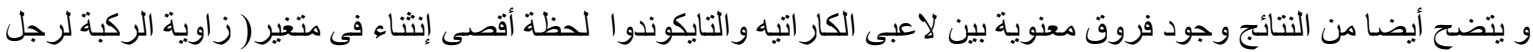

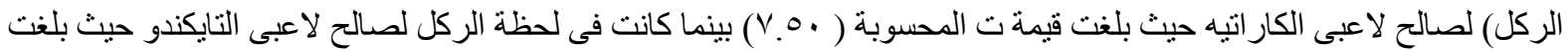

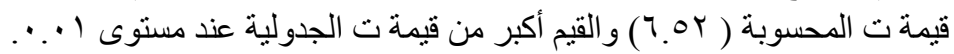

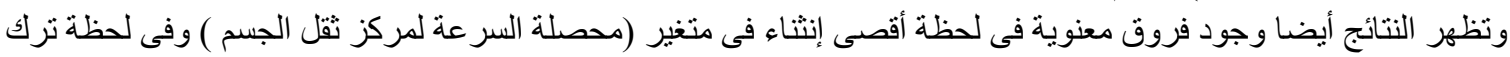

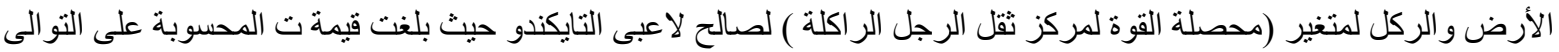

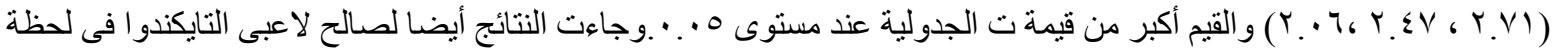

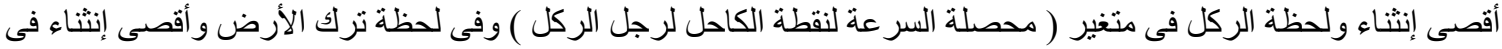

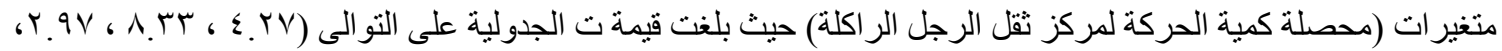

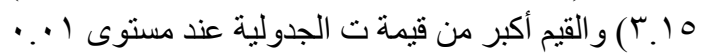

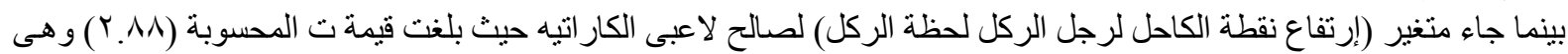

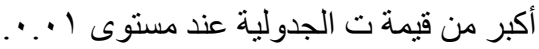

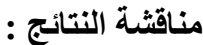

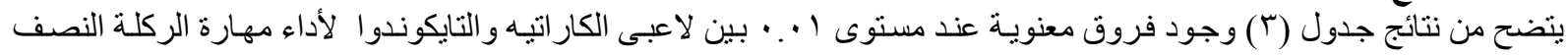

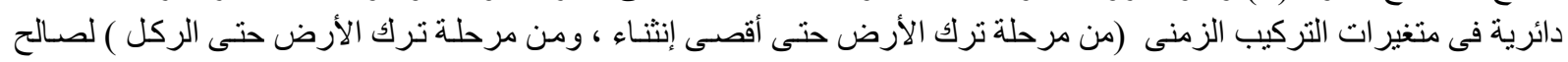

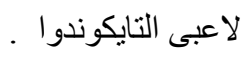

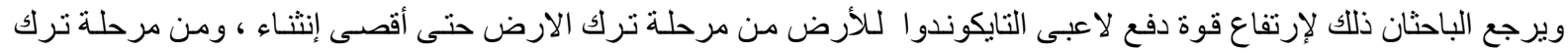

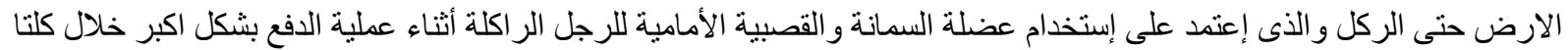

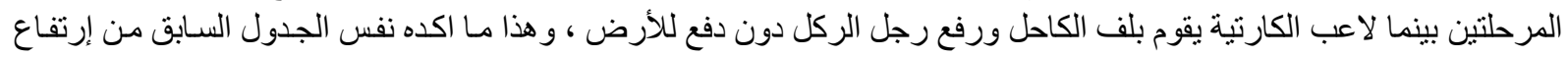

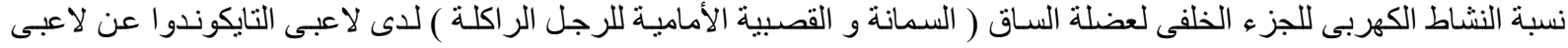

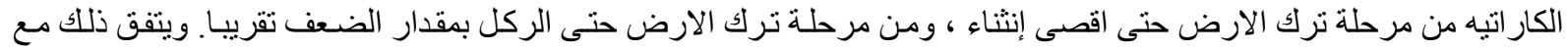

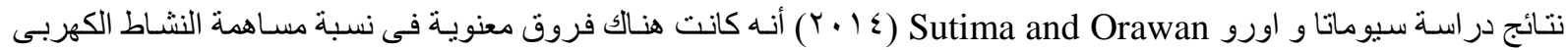

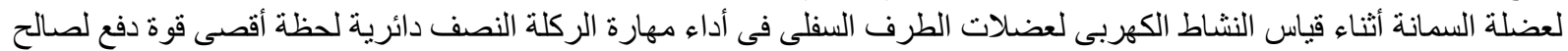

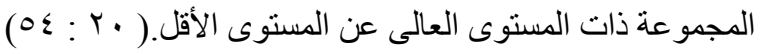

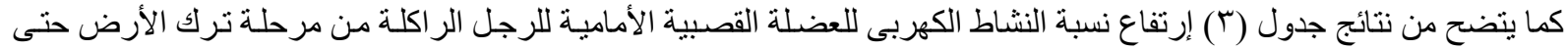

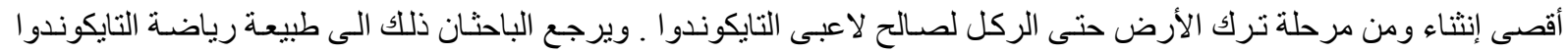

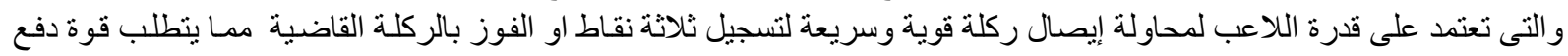

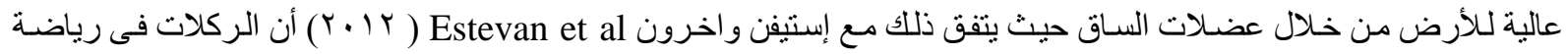

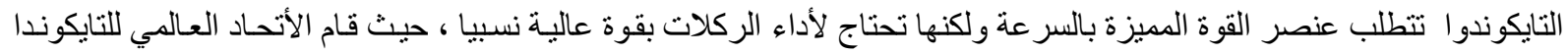

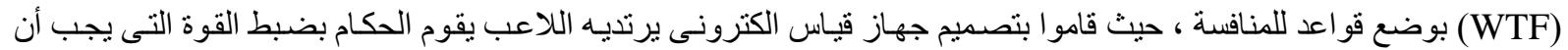

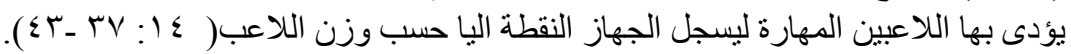

كما أظهرت نتائج جدول (r) وجود فروق الرون معنوية لصالح لاعبى الكار اتيه فى نسبة النشاط الكهربى للعضلة المستقيمة الفخذية الرباعية

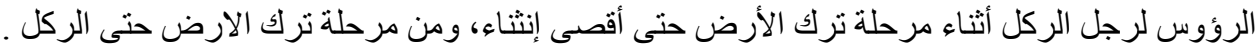

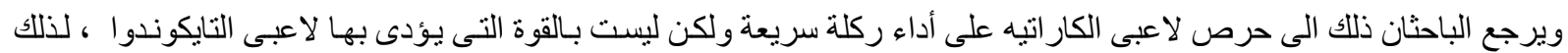

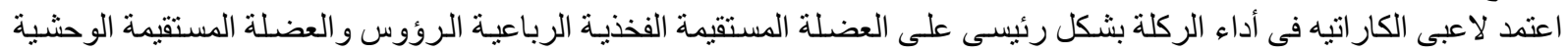

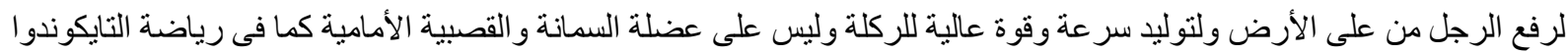
حتى يسنطيع التحكم فى قوة الركلة لفرملة الحركة فى المرحلة النهائية للمهارة حتى لالي يحصل على على إنذار .

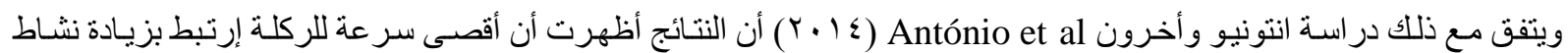

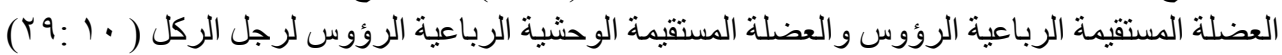

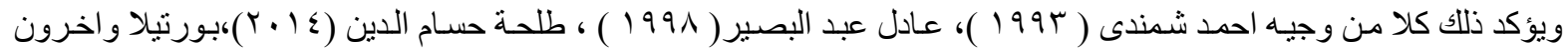
م portela et al 


\section{صبحى حسونة حسونة \\ محمد أحمد عبد الفتاح زايد}

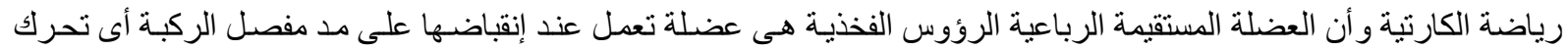

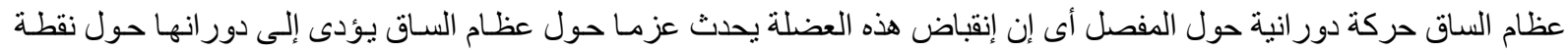

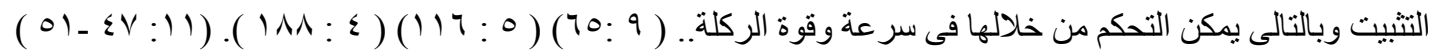

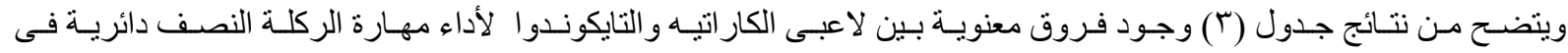
متغير المسافة بين القدمين فى وضع الأرنكاز لحظة ترك الأرض لصالح لاعبى الكار اتيه .

ويعزو الباحثان ذلك الى أن لاعب الكار اتيه يحتاج أن يكون منوازن فى وقفة إستعداده بشكل مستمر اثناء المبار اة حتى يتمكن من أداء

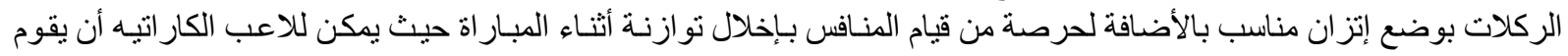

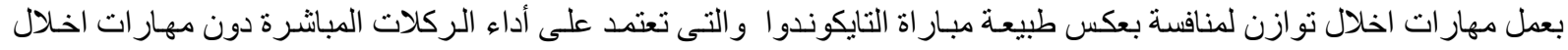

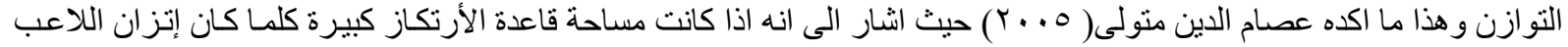

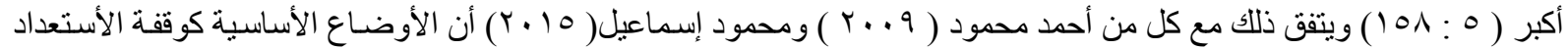

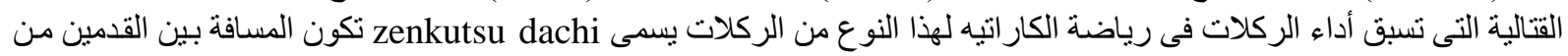

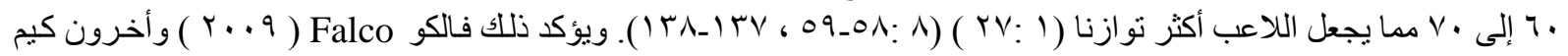

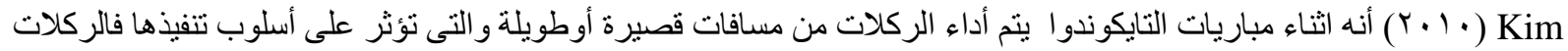

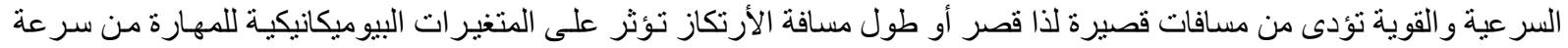

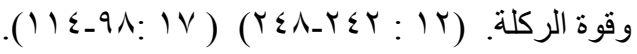

كما يتضح من نتائج جدول (r) وجود فروق معنوية لحظة اقصى إنثاء فى ( زاوية الركبة لرجل الركل لحظة أقصى إنثناء ) لصـالح

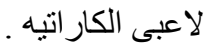

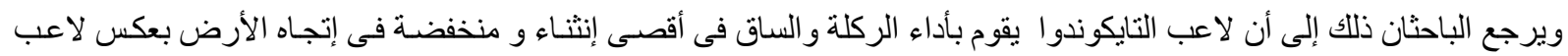

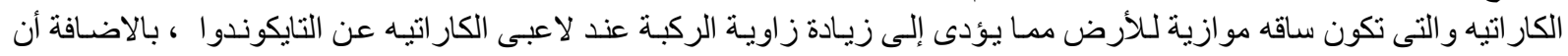

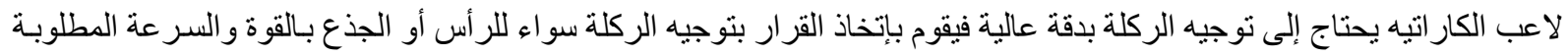

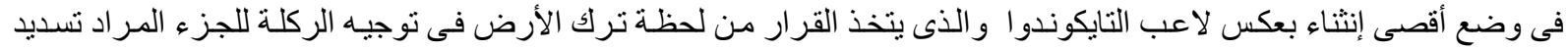

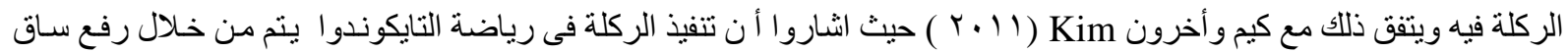

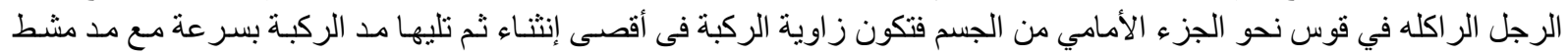

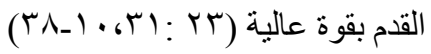

كما يتضح من نتائج الجدول (ب) أن زاوية الركبة للرجل الر اكلة لحظة الركل للاعبى الكار اتيه اقل من لاعبى التايكوندوا . ويرجع الباحثان ذلك لحرص لاعبى الكار اتيه على وصول الركلة للهدف بأقل قوة ممكنه طبقا لقانون اللعبة فيستطيع لاعبى الكار التانيه

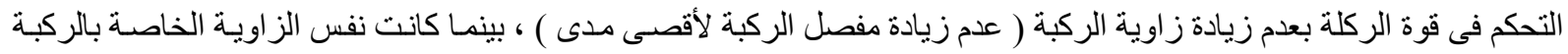

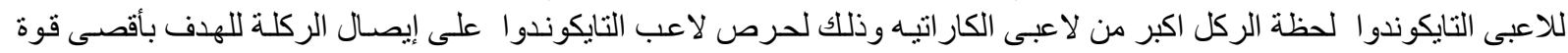

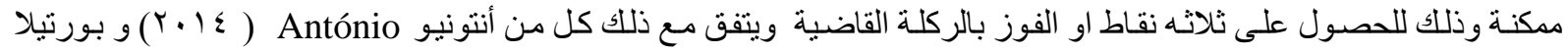

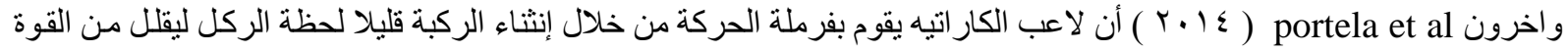

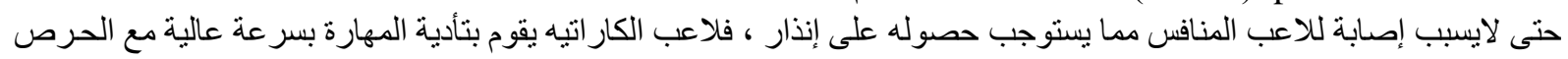

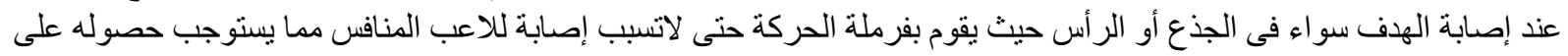

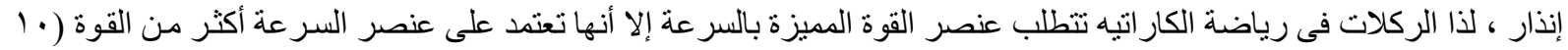

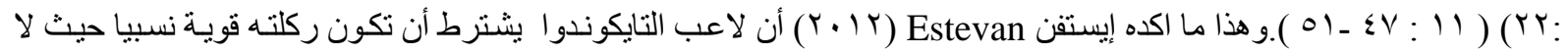

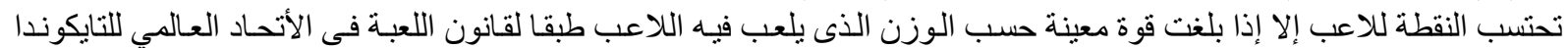

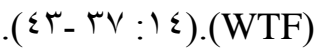

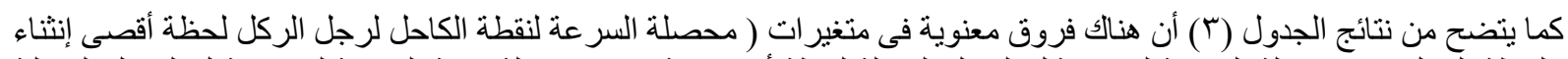

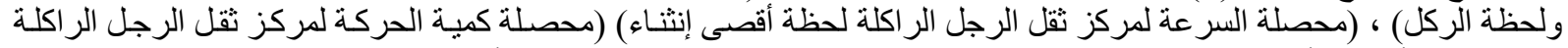
لحظة ترك الأرض و أقصى إنثاء) ، (محصلة القوة لمركز نقل الرجل الر اكلة لحظة ترك الأرض و الركل ) لصالح للاعبى التايكوندو الرا التها

ويعزو الباحثان ذلك إلى أن محصلة السرعة تزداد بزيادة محصلة القوة وكمية الحركة في بداية الركلة وأن البداية الناجحة هى التى التى

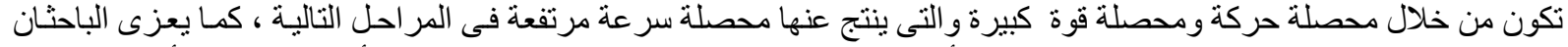

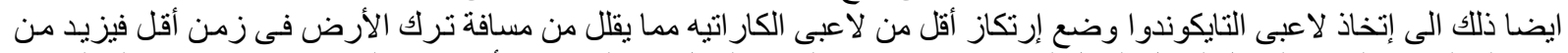

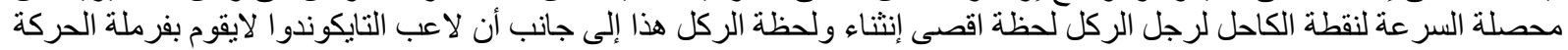




\section{صبحى حسونة حسونة}

محمد أحمد عبد الفتاح زايد

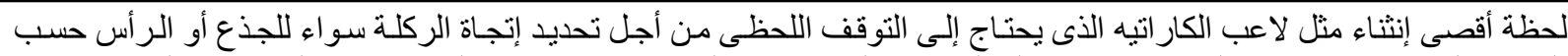

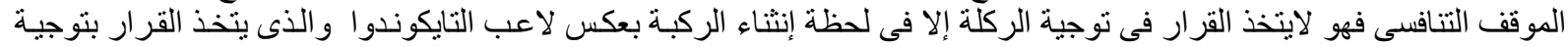

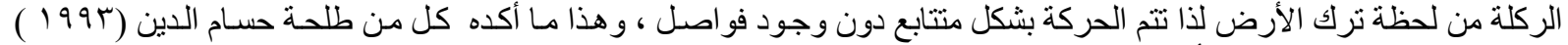

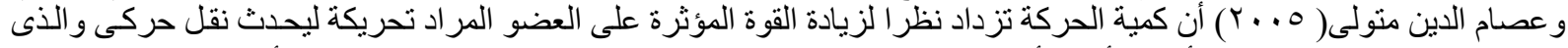

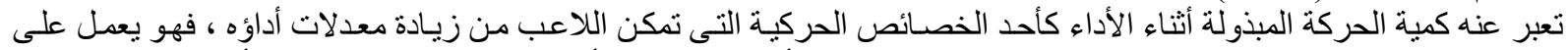

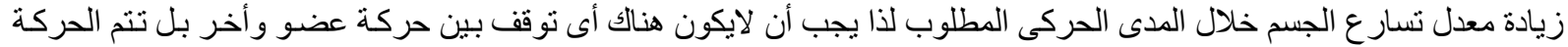

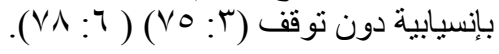

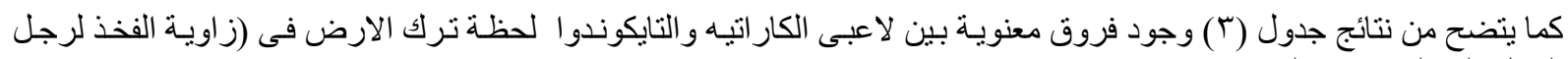

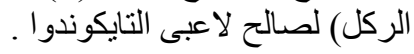

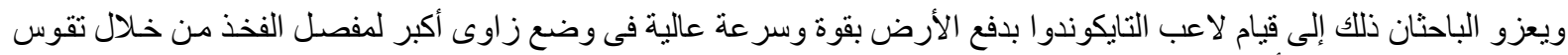

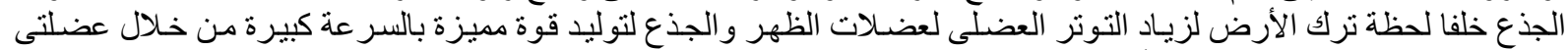

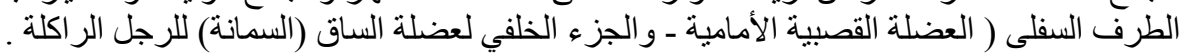

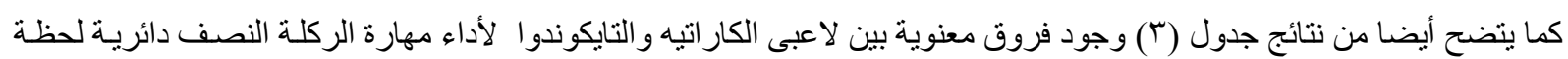

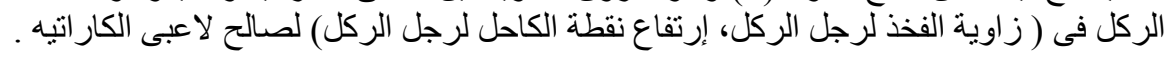

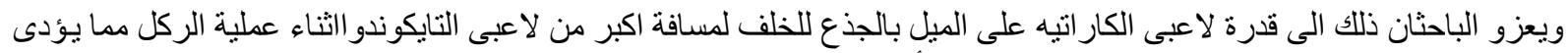

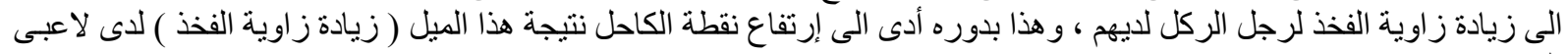
الكار اتيه.

من خلال ما تم عرضه ومناقثته استنتج الباحثان ما يأتي:

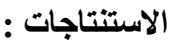

إستنتاجات خاصة بلاعبى الكار اتيه: تميز لاعبى الكاراتيه عن لاعبى التايكوندوا فى تأدية مهارة الركلة النصف دائرية من حيث الدقة وإرتفاع الركلة نظرا للأستنتاجات التالية:

- ل لاعبى الكار اتيه يؤدو ا مهارة الركلة النصف دائرية من مسافة إرتكاز كبيرة عن لاعبى التايكو ندو اللحفاظ على توازن اللاعب لذا يزيد زمن ترك اللاعب للأرض مما يزيد من زمن تأدية المهارة .

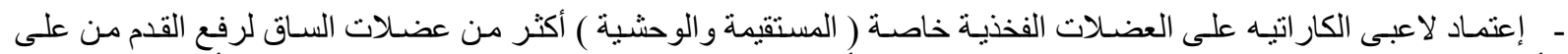

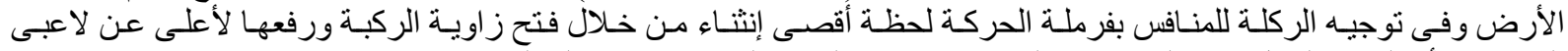

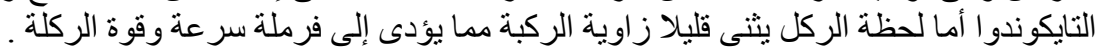

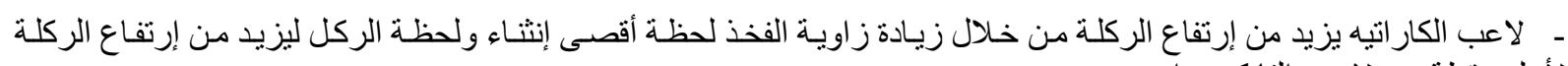
لأعلى نقطة عن لاعبى التايكوندوا

تميز لاعبى التايكوندوا عن لاعبى الكاراتيه فى تأدية مهارة الركلة النصف دائرية من حيث سرعة وقوة الركلة نظرا للأستتتاجات التالية:

- ـ لاعبى التايكوندو ايؤدو ا مهارة الركلة النصف دائرية من مسافة إرتكاز قصيرة ومتوسطة مما يعمل على تقليل زمن ترك الرجل ل للأرض مما يزيد من سرعة الركلة.

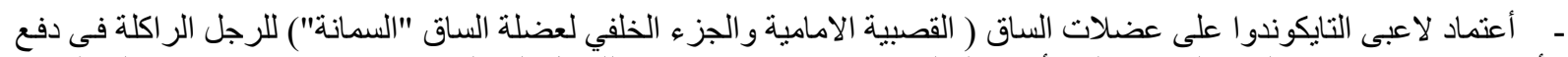

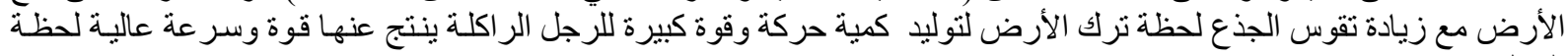

- ـ لاعبى الثايكوندوا يؤدون المهارة بدون فو اصل زمنية (عدم فرملة الحركة خلال أداء المهارة) مما يزيد من سرعة وقوة الركلة

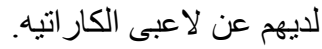




\section{صبحى حسونة حسونة}

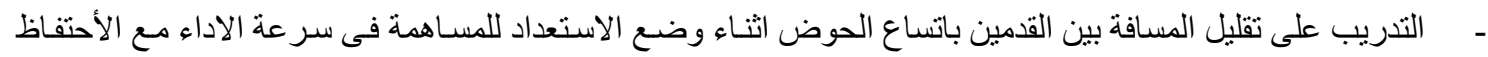

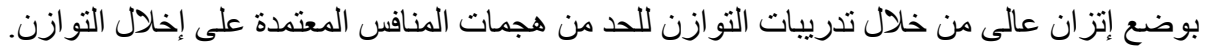

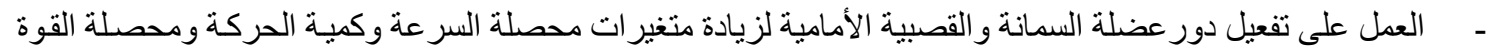

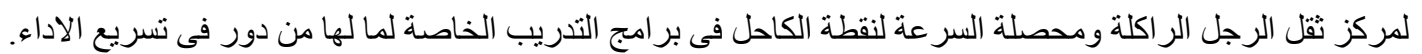

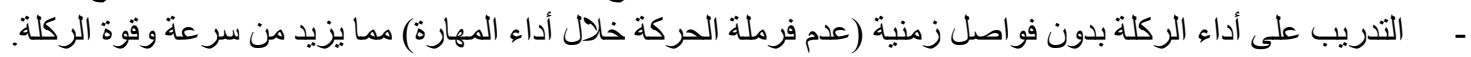

\section{توصيات للاعبى التايكوندوا :}

- العمل على تفعيل دور عضلات الفخذ الرباعية لما لها من دورفى الوصول بالركلة الى اعلى دقة وسر عة ممكنة للركلات

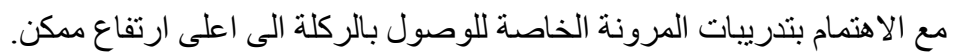

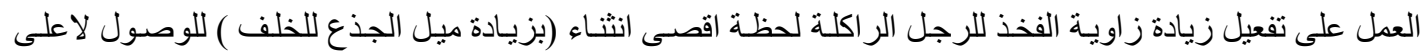

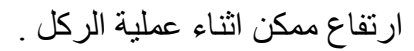
- العمل على تفعيل زيادة زاوية الركبة لحظة أقصى إنثاء ( بجعل الساق مو ازية للأرض) للتحكم فى توجيه الضربة بدقة وقوة وسر عة عالية.

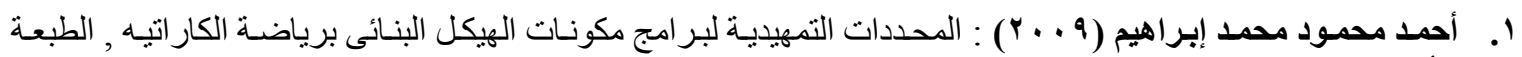

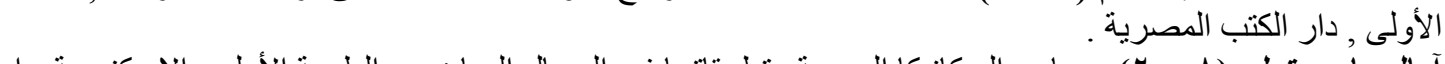

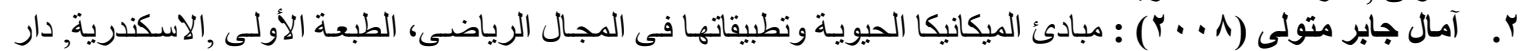

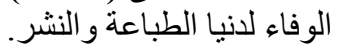

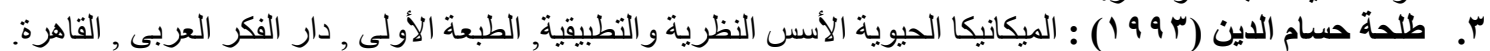

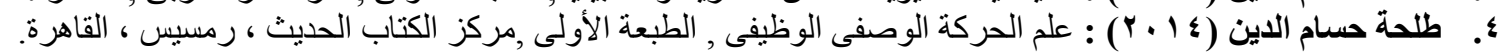

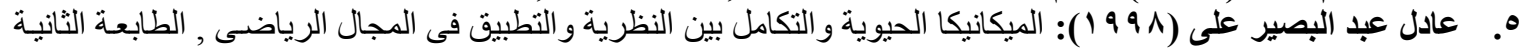

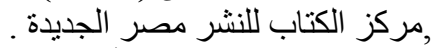

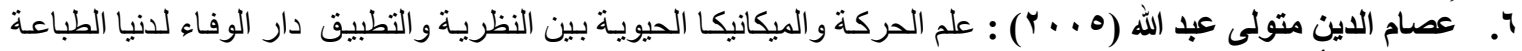
و النشر ر الأسكندرية.

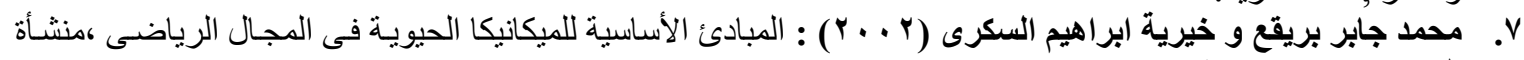
المعارف , الاسكندرية. ^. محمود إسماعيل عبد الحميد الهاشمى (10 ب ؟) : الكار اتيه , الطبعة الأولى ,مركز الكتاب الحديث للنشر رمسيس القاهرة 9. وجيه احمد شمندى (ب9 9 1 ) : الكار اتيه الحديث بين النظرية و التطبيق، مطبعة خطاب ، القاهرة. الكلمات المفتاحية : النشاط الكهربى للعضلات ، التحليل ثنائى الأبعاد ، سرعة الركلة ،قوة الركلة. 
10. António,Marco,Colaço,Renato,Cordeiro,Mári,mRodrigues,Orlando,AbelAbreu,Gonçalo(2 014): Characterization of kinesiologicalpatterns of the frontal kick,mae-geri,in karate experts and non-karate practitioners Revista de Artes Marciales Asiáticas Volumen 9(1),p.20-31.

11. Bruno Sergio, Portela,Michelle, ReginaBarbosa,Timothy Gustavo Cavazzotto,Marcus Peikriswili Tartaruga (2014) : Kinematics analysis of the front kick with and without impact on traditional karate, $\mathbf{1 0}$, p. 47-51.

12. Falco, Alvarez, Castillo, Estevan,Martos, Mugarra, and Iradi (2009) : Influence of the distance in a roundhouse kick's execution time and impact force in Taekwondo. J Biomech $\mathbf{4 2}$, p.242-248.

13. Isaac Estevan, Octavio a' Ivarez, Coral Falco, Javier molina-garci'a, and Isabel Castillo (2011): Impact force and time analysis influenced by execution distance in a roundhouse kick to the head in taekwondo. J Strength Cond Res, 25(10), p.28.

14. Isaac Estevan, Coral Falco, Octavio Álvarez, Javier Molina-García (2012): Effect of olympic weight category on performance in the roundhouse kick to the head in taekwondo. $\mathbf{J}$ Hum Kinet, 31: p. 37-43.

15. Isaac Estevan, and C. Falco (2013): Mechanical analysis of the roundhouse kick according to height and distance in taekwondo. Biol Sport, 30(4): p. 275.

16. Jacek Wąsik (2011): Kinematics and Kinetics of Taekwon-do Side Kick. J Hum Kinet, 30: p. 13-20.

17. Jae-Woong Kim, Moon-Seok Kwon, Sree Sushma Yenuga, \&Young-Hoo kwon (2010): The effects of target distance on pivot hip, trunk, pelvis, and kicking leg kinematics in Taekwondo roundhouse kicks. Sports Biomech, 9(2): p. 98-114.

18. Sherif Ali Taha1, Abdel-Rahman Ibrahim Akl, Mohamed Ahmed Zayed (2015): Electromyographic Analysis of Selected Upper Extremity Muscles during Jump Throwing in Handball. American Journal of Sports Science, 3(4): p. 79.

19. Sillanpaa,J (2007): Electromyography for Assessing Muscular Strain in the Workplace, Finnish Institute of Occupational Health, People and Work, Research, p.79.

20. Sutima Thibordeeand and Orawan Prasartwuth (2014): Factors Influencing the Impact Force of the Taekwondo Roundhouse Kick, CMU. J. Nat. Sci, Vol13, p. 54.

21. Thibordeeand, and Prasartwuth (2014): Factors Influencing the Impact Force of the Taekwondo Roundhouse Kick. Chiang Mai University Journal of Natural Sciences, P.13.

22. Tomasz Halski, Piotr Żmijewski, Paweł Cięszczyk, Barbara Nowak Kuba Ptaszkowski, Lucyna Slupska1, Robert Dymarek, Jakub Taradaj(2015): Electromyographic Analysis of the Hip Extension Pattern in Visually Impaired Athletes. J Hum Kinet,48: p. 53-6.

23. Young Kwan Kim, Yoon Hyuk Kim, and Shin Jaim (2011): Inter-joint coordination in produc-ing kicking velocity of Taekwondo kicks. Journal of Sports Science and Medicine 10, p. 31-38. 


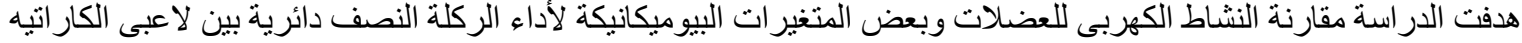

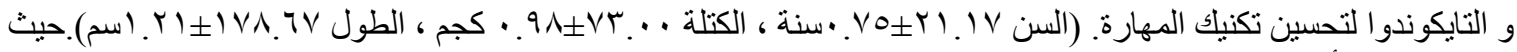

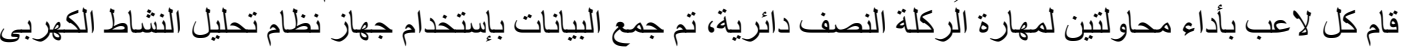

(MegaWin ME6000)

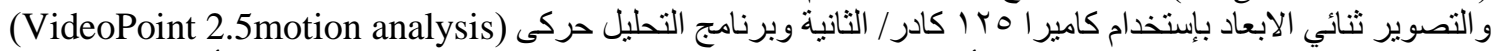

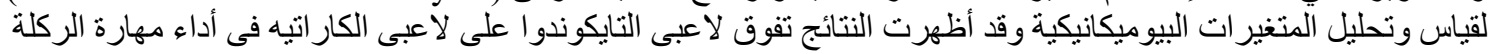

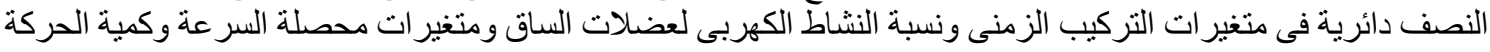

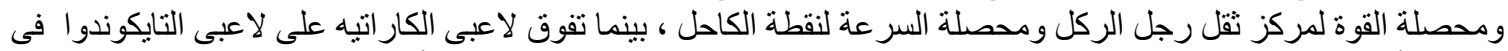

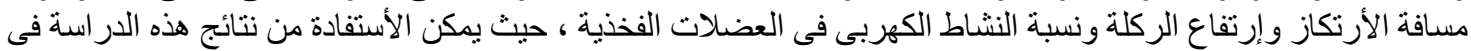

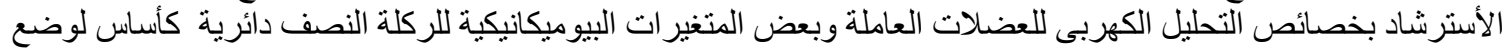

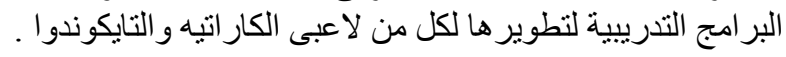

\section{الملخص باللغة الانجليزية :}

The study aimed to compare the electrical activity of the muscles and some biomechanical variables for the performance of the half-circular kick between karate players and taekwondo to improve skill technique. (Age $21.17 \pm 0.75$ years, mass $73.00 \pm 0.98 \mathrm{~kg}$, length $178.67 \pm 1.21 \mathrm{~cm}$ ). Each player performed two semi-circular kickballs, the data was collected using the MegaWin ME6000 system. The results showed that the taekwondo players were superior to the karate players in the performance of the semi-circular ball skill in the temporal variables and the percentage of activity. Electrocardiogram Leg and velocity variables and the amount of movement and strength of the center of the kicking man and the speed of the ankle point, while the karate players exceeded the taekwondo players in the distance of the base and the height of the kick and the percentage of electrical activity in the femoral muscles, where the results can be used in this study guided by the characteristics of electrical analysis of working muscles And some biomechanical variables of the semi-circular kick as a basis for developing training programs for the development of both karate and taekwondo players 